\title{
Geriatric Medicine, an Underrecognized Solution of Precision Medicine for Older Adults in Korea
}

With unprecedented rapid population aging and social structural remodeling, care needs for older adults are skyrocketing in a multifaceted fashion; this includes the healthcare and social welfare sectors in Korea. Since population aging and soaring medical costs exist as unified issues in both developed and developing countries around the world, societies have tried to find solutions to care older adults in optimized way, with increasing cost effectiveness and also keeping qualities of care uncompromised. Therefore, when preparing solutions for soaring healthcare needs in older population, physicians, policymakers, and public health professionals in Korea can borrow from the experiences of other countries to predict the pros and cons of various healthcare models that can be implemented in the Korean healthcare system in the near future.

In terms of healthcare models to optimize complex care issues in older adults with multimorbidity, frailty, and functional impairment, the concept of geriatric medicine has been widely recognized as a necessary solution in countries with varying healthcare delivery systems. Take apart from the United Kingdom's National Health Service that has traditionally focused on cost-effectiveness and has fostered geriatric medicine as a core specialty in serving healthcare needs of older adults, now we reckon that even the United States, which employs a traditional extreme of a fee-for-service system, is endeavoring to improve their healthcare system's efficacy by leveraging geriatric medicine. ${ }^{1)}$

The concept of geriatric medicine starts from recognizing older patients as individuals with vast heterogeneity in terms of disease burden and physiological and functional reserve, even among those in the same age group. Moreover, similar to approaches observed in pediatrics, which begins by noticing physiological differences between pediatric and adult populations, geriatricians note that geriatric patients are not just "older" adults with higher chronological age. For example, when initiating therapeutic measures for a clinical condition, geriatricians understand that riskbenefit assumptions produced in clinical trials performed in younger adults are often useless in multimorbid, frail, and functionally impaired older patients. ${ }^{2)}$ In this manner, geriatric viewpoints try to incorporate coexisting chronic diseases, social resources, and physical performance in making patient-centered clinical decisions.

In these geriatric patients, care needs are higher, and longitudinal caregiving processes frequently involve different levels of care, including tertiary hospitals, subacute care, and community-based long-term care in patients' homes or nursing facilities. ${ }^{3)}$ To seamlessly bridge gaps between different clinical settings, the geriatric concept of care places the patient at the center of these transitions. Furthermore, to cover multiple chronic conditions, these patients are often served by doctors of varying specialties, resulting in an increased probability of exposure to polypharmacy and potentially inappropriate medications for older adults. ${ }^{4)}$ However, treatment strategies concerning these complex conditions can be reconciled using a patient-centered geriatric approach, including comprehensive geriatric assessment. With these comprehensive approaches, geriatrics can provide precision medicine to meet specific personalized needs based on multifaceted risk assessment for older patients; this can be accomplished even without performing high-cost laboratory tests using modern -omics methods or high-tech medical devices. ${ }^{5)}$

However, unlike other developed countries that have tried to provide patient-centered, continued care for patients from acute hospitalization to community-based chronic care by incorporating the concept of geriatric medicine, in Korea, the concept of geriatric medicine exists only in a few hospitals. For example, although the Long-Term Care Insurance (LTCI) program began in 2008 to serve older adults with functional impairment, the concept of geriatric medicine was not incorporated in this program. In contrast to similar care programs in other developed countries, the Korean LTCI program does not cover medical illness and subacute to chronic medical care in patients with functional impairment; this results in many older patients with multiple chronic diseases and functional impairment end up being admitted to chronic care hospitals, where they remain hospitalized until their death. ${ }^{6)}$ Additionally, although comorbidity, frailty, and functional decline are overlapping geriatric conditions in older adults, ${ }^{7)}$ when patients are admitted to chronic care hospitals, nursing service is not covered by the LTCI. Reciprocally, in older patients who reside in nursing homes because of care needs, medical service is covered by the National Health Insurance (NHI) service rather than by the LTCI.

Furthermore, in The Plan for Community Care from the Ministry of Health and Welfare that proposed this year to 
solve problems associated with a fractionized care system in care transitions, we note that the concept of geriatric medicine is still widely lacking. In addition, the concept of geriatric medicine such as deprescribing or reconciliation of medicines is still nonexistent, and this value-based care is not yet reimbursed by the Korean NHI service. In contrast with other aging societies that try to focus on valuebased care while maintaining or reducing the total amount of medical services for older adults, the Korean NHI system maintains a strong focus on reducing the fee for each service. Predictably, in response to this cost-reducing approach, physicians consistently endeavor to maximize medical service volume to sustain revenue stream even in the circumstance of decreasing fees for medical services. From geriatric point of view, this "chicken game" will be never sustainable, with a vast amount of healthcare resources being used on inappropriate treatments for frail older people, will decrease quality of care, and threat the long-triumphed cost-effectiveness of the Korean NHI system in terms of healthcare outcomes.

With an accelerating trend of population aging, the gap between unmet needs for geriatric medicine and the current fee-for-service-based care system will continue to grow. However, the Korean government and NHI system still remain complacent, exhibiting reluctance to incorporate the concept of geriatric medicine. Unlike the vast majority of countries where geriatric medicine has been a well-recognized specialty or subspecialty, ${ }^{8,9)}$ controversies regarding whether geriatric medicine specialists will eventually be required in Korea remain. However, global evidence advocating the patient-centered, geriatric approach to care is ever increasing; thus, to prevent an aging-related catastrophe that we expect in the next decade, the Korean government should note that the current healthcare system should adopt the concept of clinical geriatrics as soon as possible.

\section{CONFLICTS OF INTEREST DISCLOSURES}

The authors declare no conflicts of interest.

\section{REFERENCES}

1. Applegate WB, Ouslander JG, Kuchel GA. Implementing “patient- centered care": a revolutionary change in health care delivery. J Am Geriatr Soc 2018;66:1863-5.

2. Kojima T. The need for actions against polypharmacy in older people with frailty. Ann Geriatr Med Res 2018;22:111-6.

3. Kim CO. Time to conduct community-oriented researches in geriatrics. Ann Geriatr Med Res 2018;22:109-10.

4. Kim MY, Etherton-Beer C, Kim CB, Yoon JL, Ga H, Kim HC, et al. Development of a consensus list of potentially inappropriate medications for Korean older adults. Ann Geriatr Med Res 2018;22:121-9.

5. Williams GR. Geriatric assessment: precision medicine for older adults with cancer. J Oncol Pract 2018;14:97-8.

6. Ga H, Won CW, Jung HW. Use of the frailty index and FRAIL-NH scale for the assessment of the frailty status of elderly individuals admitted in a long-term care hospital in Korea. Ann Geriatr Med Res 2018;22:20-5.

7. Jung HW, Jang IY, Lee YS, Lee CK, Cho EI, Kang WY, et al. Prevalence of frailty and aging-related health conditions in older Koreans in rural communities: a cross-sectional analysis of the aging study of Pyeongchang rural area. J Korean Med Sci 2016;31:34552.

8. Reiter R, Diraoui S, Van Den Noortgate N, Cruz-Jentoft AJ. How to become a geriatrician in different European countries. Eur Geriatr Med 2014;5:347-51.

9. Kim MY, Subramaniam P, Flicker L. The Australian aged care and its implications for the Korean aging crisis. Ann Geriatr Med Res 2018;22:9-19.

Hee-Won Jung ${ }^{1}$, Jae-Young Lim $^{2}$

${ }^{1}$ Graduate School of Medical Science and Engineering, Korea Advanced Institute of Science and Technology (KAIST), Daejeon, Korea ${ }^{2}$ Department of Rehabilitation Medicine, Seoul National University Bundang Hospital, Seoul National University College of Medicine, Seongnam, Korea

Corresponding Author: Hee-Won Jung, MD Graduate School of Medical Science and Engineering, Korea Advanced Institute of Science and Technology (KAIST), Daejeon, Korea Tel: +82-42-350-4283, Fax: +82-42-350-4269, E-mail: dr.ecsta@gmail.com ORCID code: https://orcid.org/0000-0002-2583-3354 